\title{
CORRECTION
}

\section{Correction: Functional brain networks in never-treated and treated long-term Ill schizophrenia patients}

Li Yao, Fei Li (iD, Jieke Liu, Wei Liao (D, Xiaojing Li, Mingli Li, Yajing Meng (D), Sugai Liang, Chengcheng Zhang, Xiao Yang, Qiang Wang, Xiaohong Ma, Wanjun Guo, John A. Sweeney, Qiyong Gong, Su Lui, Wei Deng and Tao Li

Neuropsychopharmacology (2020) 45:1406; https://doi.org/10.1038/s41386-020-0673-4

Correction to: Neuropsychopharmacology https://doi.org/10.1038/ s41386-019-0428-2, published online 4 June 2019

The original version of this Article had an error in the Funding and Disclosure section. The grant number for the National Key
Research \& Development Program of China was incorrectly given as Grant no. 2016YFC1201700. The correct number is Grant no. 2016 YFC1307200. 